\title{
MIR130B Pre-miRNA
}

National Cancer Institute

\section{Source}

National Cancer Institute. MIR130B Pre-miRNA. NCI Thesaurus. Code C82764.

MIR130B is an oligoribonucleotide that is encoded by the MIR130B gene and is involved

in the regulation of gene expression. 